\title{
Large Immunologic Species of Human Chorionic Gonadotropin in Placental Extracts
}

\author{
Shimpei TOJO, Yoshihiko ASHITAKA, TAKeshi MARUO \\ and Masanobu OHASHI \\ Department of Obstetrics and Gynecology, Kobe University \\ School of Medicine, Kobe 650, Japan
}

\begin{abstract}
Synopsis
Gel filtration of extracts of human chorionic tissues cultivated in vitro and of culture media has been performed. Heterogeneous immunoreactive forms of hCG and its subunits in tissue extracts were apparent. A void-volume immunoreactive peak of hCG was identified in extracts of chorionic tissues cultivated for a very short period. The immunoreactive void-volume material, which did not readily convert to the authentic form of hCG either spontaneously in refiltration or in $5 \mathrm{M}$ guanidine, could not be distinguished immunologically from purified hCG and yielded an hCG-like component in trypsinization.

On gel filtration of chorionic tissue extracts, radioreceptor activity for hCG utilizing membrance fraction of pseudopregnant rat ovary always demonstrated a single peak in the region of ${ }^{125} \mathrm{I}-\mathrm{hCG}$ used as column marker, indicating high receptor activity in the authentic form of hCG and low in the large immunologic species of hCG. Heterogeneity in the molecular size of bicactive hCG was not found with radioreceptor assay.

On the other hand, gel filtration profiles of chorionic tissue extracts and of culture media showed that the large immunologic species of hCG is a predominant form of the hormone in the chorionic tissue cultivated for a short period and the authentic form of $\mathrm{hCG}$ is a predominant form in the media. These data suggest that immunoreactive "big hCG" synthesized initially in placenta may be converted to the authentic form of hCG by trypsin-like enzyme and that the authentic form of hCG may be readily secreted into the media. These results support the proposal that hCG is also synthesized as prohormone in placenta. However, the biosynthetic process of hCG subunits remains yet to be resolved.
\end{abstract}

Radioimmunologic evidences accumulated in recent years suggest that glycoprotein hormons within the endocrine glands exhibit molecular heterogeneity (Vaitukaitis, 1974; Prentice \& Ryan, 1975; Maruo, 1976). A previous report (Maruo, 1976) on studies of the mechanism of human chorionic gonadotropin (hCG) biosynthesis has provided evidence for the existence of hCG immunoreactive material larger than the wellcharacterized urinary hCG. The large

Received February 10, 1977. immunologic species of $\mathrm{hCG}$ detected in extracts of chorionic tissues cultivated in vitro for a short period was eluted in the void volume of a Sephadex G-100 column. This immunoreactive void-volume material also contained components reacting in the anti-hCG- $\alpha$ system and in the anti-hCG- $\beta$ system. It has been simultaneously suggested by radioactive amino acid incorporation studies that the immunoreactive voidvolume material seemed to play a precursor role in hCG biosynthesis.

Finally, recent availability of the radio- 
receptor assay for hCG has permitted an assessment of biological activity of various species of hCG of apparently different molecular size obtained following gel filtration. The present study was undertaken to investigate the characteristics of the immunoreactive void-volume material and its relation to $\mathrm{hCG}$ and to search for the heterogeneity of hCG with respect to biological activity, using the radioreceptor assay.

\section{Materials and Methods}

\section{Chorionic tissue culture}

Human chorionic tissues were obtained at the time of therapeutic abortion in the first trimester of pregnancy. Organ culture of chorionic tissues was carried out in the same manner as previously described (Maruo et al., 1974). Incubations were performed for varying time periods at $37^{\circ} \mathrm{C}$ under a $95 \%$ air $-5 \% \mathrm{CO}_{2}$ atmosphere in the synthetic medium " 199 " containing penicillin $(100 \mathrm{U} / \mathrm{ml})$ and streptomycin $(100 \mu \mathrm{g} / \mathrm{m} l)$.

At the end of culture, tissues and media were separated. A total of $500 \mathrm{mg}$ of the cultured chorionic tissues was homogenized in $3 \mathrm{~m} l$ distilled water and centrifuged at $10,000 \times g$ for $10 \mathrm{~min}$ at $4^{\circ} \mathrm{C}$. The supernatant was then subjected to Sephadex gel filtration. The pooled media were dialyzed at $4^{\circ} \mathrm{C}$, lyophilized, dissolved in $3 \mathrm{~m} l$ distilled water and then subjected to gel filtration.

\section{Sephadex gel filtration}

A $90 \mathrm{~cm} \times 2 \mathrm{~cm}$ Sephadex G-100 column (Phrmacia) was equilibrated at $4^{\circ} \mathrm{C}$ with $0.005 \mathrm{M}$ Tris- $\mathrm{HCl}$ buffer, $\mathrm{pH}$ 8.6. The void volume of the column was determined with blue dextran. The column was standardized with highly purified urinary $\mathrm{hCG}, \mathrm{hCG}-\alpha$ and hCG- $\beta$, labeled with ${ }^{125} \mathrm{I}$, as described previously (Ashitaka et al., 1974). Fractions of $3.5 \mathrm{ml}$ were collected at a flow rate of approximately $17 \mathrm{ml} / \mathrm{hr}$. Placental extracts and culture media were independently applied to the column and eluted with the same buffer. The tubes containing immunoreactive void-volume material were combined and concentrated by lyophilization for refiltration or further characterization.

\section{Radioimmunoassays of $h C G$ and its subunits}

Each eluate was radioimmunoassayed for hCG, hCG- $\alpha$ and hCG- $\beta$, using the respective homologous assay system which has been previously described (Ashitaka et al., 1974). The urinary purified hCG $(15,000 \mathrm{IU} / \mathrm{mg}$, by an ovarian weight method $)$ pre- pared as described previously (Ashitaka et al., 1970) was separated into subunits in a manner similar to that described by Swaminathan and Bahl (1970). Iodination of these glycoproteins was carried out by the lactoperoxidase method with $\mathrm{Na}^{125}$ I (Ashitaka and Koide, 1974). The average specific radioactivity of ${ }^{125} \mathrm{I}$-hCG was $66-74 \mu \mathrm{Ci} / \mu \mathrm{g},{ }^{125} \mathrm{I}-\mathrm{hCG}-\alpha: 80 \mu \mathrm{Ci} / \mu \mathrm{g}$, ${ }^{125} \mathrm{I}-\mathrm{hCG}-\beta: 60 \mu \mathrm{Ci} / \mu \mathrm{g}$. For these studies the real amounts of these hormones were calculated by subtracting the interferences of non-homologous proteins in the homologous assays since highly purified hCG exhibited approximately $1 \%$ cross reactivity in the homologous hCG- $\alpha$ system and approximately 10\% in the homologous hCG- $\beta$ system (Ashitaka et al., 1974).

\section{Radioreceptor assay}

In some experiments the effluent fractions were simultaneously measured for hCG bioactivity by the radioreceptor assay in a similar manner as described by Lee and Ryan (1973).

$2,000 \times g$ fractions of ovarian homogenate from pseudopregnant rat (Sprague-Dawley strain, Nihon CLEA Co., Japan) primed with PMS and hCG were used as receptor preparation for hCG. For assays $0.5 \mathrm{ml}$ of $40 \mathrm{mM}$ Tris- $\mathrm{HCl}$ buffer of $\mathrm{pH} 7.4$ containing $0.1 \%$ bovine serum albumin (BSA), $0.1 \mathrm{ml}$ of standard or unknown samples in the same buffer, $0.1 \mathrm{~m} l$ of ${ }^{125} \mathrm{I}-\mathrm{hCG}(30,000 \mathrm{cpm})$ and finally $0.1 \mathrm{~m} l$ of $2,000 \times g$ fraction of appropriate dilution (equivalent to $2.5 \mathrm{mg}$ of wet ovary) were added to make a final volume of $800 \mu l$ per tube. The tubes were then shaken vigorously and incubated at $35^{\circ} \mathrm{C}$ for $1 \mathrm{hr}$. Following incubation, the reaction was stopped by adding $1.0 \mathrm{ml}$ of ice-cold Tris- $\mathrm{HCl}$ buffer containing $0.1 \%$ BSA. After centrifugation at $4,000 \mathrm{rpm}$ for $30 \mathrm{~min}$, the supernatant was drained and the tip of each tube was dried. The pellet remaining at the bottom of the tube was counted in an automatic gamma spectrometer.

\section{Guanidine treatment}

Lyophilized eluate containing immunoreactive void-volume material $(400 \mu \mathrm{g})$ was incubated at $23^{\circ} \mathrm{C}$ for $3 \mathrm{hr}$ in $5 \mathrm{M}$ guanidine (Nakarai Chemical Ltd., Japan) and then refiltered over the same column. All fractions from the column were dialyzed to remove guanidine and then subjected to radioimmunoassays.

\section{Tryptic digestion}

Lyophilized eluate containing immunoreactive void-volume material $(400 \mu \mathrm{g})$ was incubated with trypsin (Sigma Chemical Co., U.S. A., $100 \mu \mathrm{g} / \mathrm{m} l$ ) for $1 \mathrm{~min}$ at $37^{\circ} \mathrm{C}$. The mixture was then reacted withsoy bean trypsin inhibitor (Sigma Chemical Co., $100 \mu \mathrm{g} / \mathrm{m} l)$ and then the entire reaction mixture was subjected to the Sephadex gel filtration. 


\section{Results}

When the extract of chorionic tissue previously incubated in the synthetic medium "199" for $15 \mathrm{~min}$ was subjected to gel filtration on Sephadex G-100, hCG immunoreactivity showed a single peak immediately following the void volume of the column (Fig. 1). For the purpose of identification, the immunoreactive component appearing near the void volume was referred to as immunoreactive void-volume material in this study. The lyophilized immunoreactive void-volume material $(400 \mu \mathrm{g})$ was refiltrated on the same column to determine whether spontaneous dissociation occurred. As shown in Fig. 2, hCG immuoreactivity showed a single peak in the void volume, indicating the absence of such dissociation into the authentic form of hCG.

\section{Guanidine treatment}

The possibility that the immunoreactive void-volume material represented either a

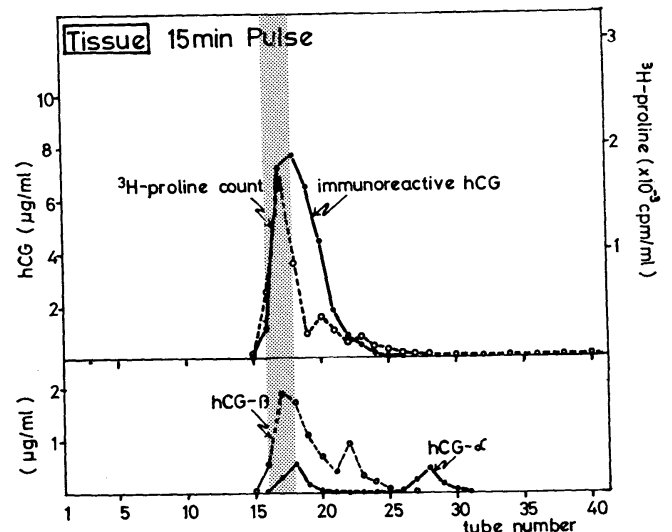

Fig. 1. Elution profiles of the placental extract on Sephadex G-100 upward column. Chorionic tissue $(100 \mathrm{mg})$ incubated in $\mathrm{M}-199(15 \mathrm{ml})$ for $15 \mathrm{~min}$ at $37^{\circ} \mathrm{C}$ was homogenized and centrifuged at $10,000 \times g$ for $10 \mathrm{~min}$. The supernatant was subjected to the column. The void volume of the column was $59.5 \mathrm{ml}$ (tube \#17) and shown in the stippled area. polymer of hCG or hCG associated with another protein was investigated by incubating it in $5 \mathrm{~m}$ guanidine prior to Sephadex gel filtration. The elution pattern of the guanidine-treated material neither showed any shift of elution position of hCG immunoreactivity nor revealed the other peak of hCG immunoreactivity smaller than a peak detected in the void volume. However, a slight decrease in hCG immunoreactivity was observed and two distinct peaks of immunoreactive hCG- $\alpha$ and hCG- $\beta$, which were respectively eluted preceding the expected position of urinary purified $\mathrm{hCG}-\alpha$ and hCG- $\beta$ used as column markers, were apparent (Fig. 2).

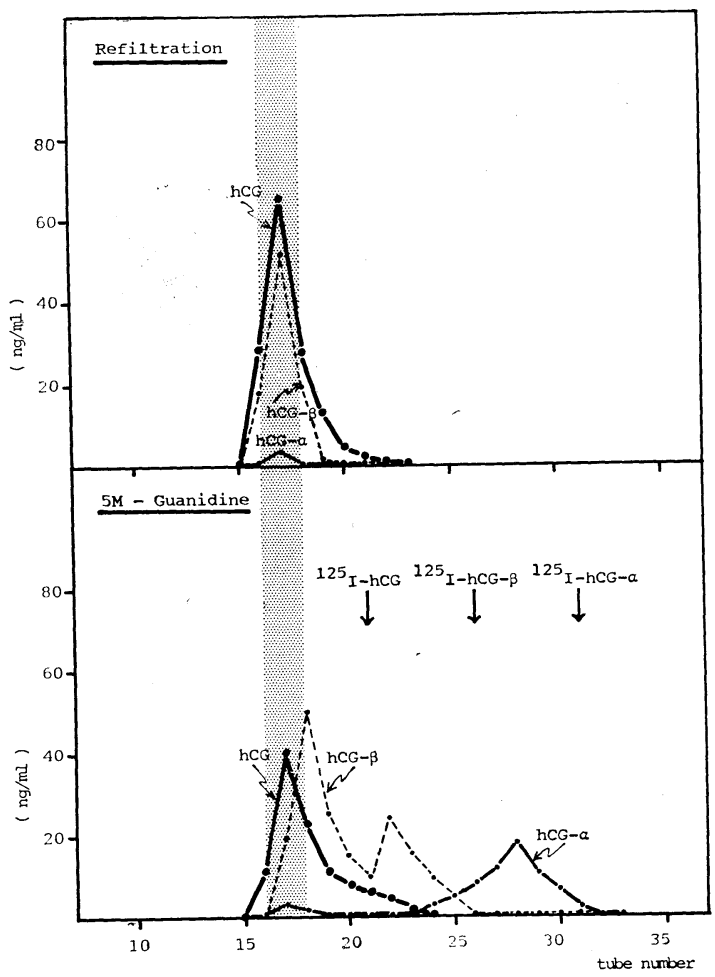

Fig. 2. Sephadex G-100 elution profile of immunoreactive void-volume material obtained from the initial gel filtration of the placental extract. The top panel shows elution profile of the immunoreactive void volume material after refiltration on the same column, employing the same conditions. The bottom panel displays elution profile after incubation in $5 \mathrm{M}$ guanidine and refiltration on the same column. 
Comparison of $h C G$ and its subunits with the immunoreactive void-volume material by radioimmunoassays

Comparison of some of the immunologic determinants of the immunoreactive voidvolume material, $\mathrm{hCG}$ and its subunits were made by determinating inhibition lines in the respective homologous radioimmunoassays for hCG, hCG- $\alpha$ and hCG- $\beta$. The standard inhibition line in each system was obtained with urinary purified hCG, hCG- $\alpha$ and $h C G-\beta$, respectively. The immunoreactive void-volume material produced the

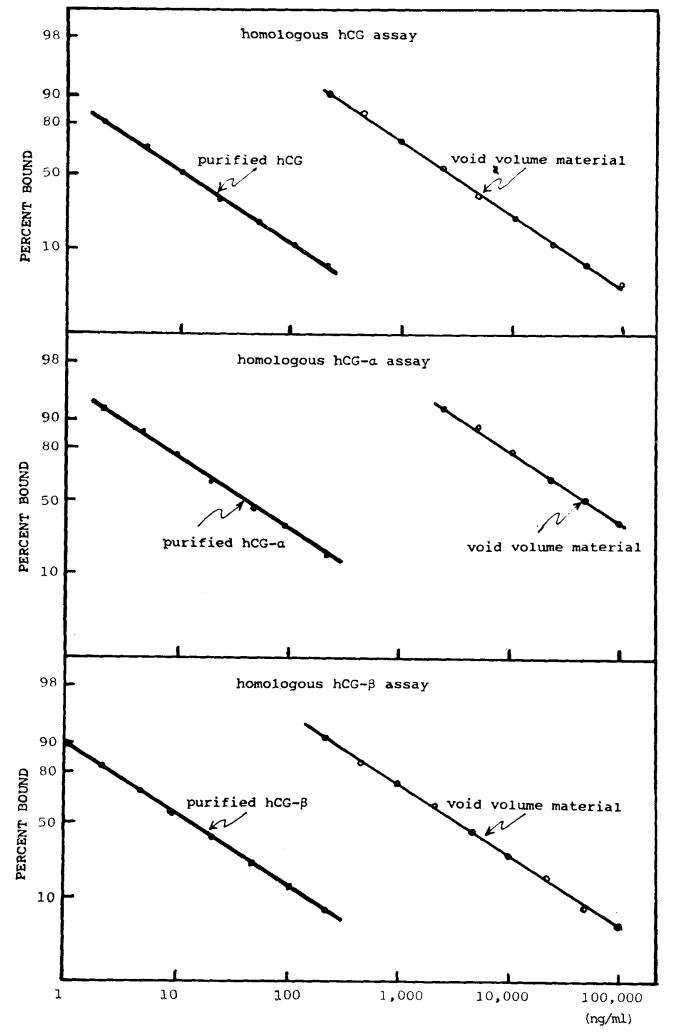

Fig. 3. Comparison of hCG and its subunits with the immunoreactive void-volume material obtained from Sephadex gel filtration of the placental extract by radioimmunoassays. The top panel represents the inhibition dose-response curves obtained in the homologous hCG system, the middle panel in the homologous hCG- $\alpha$ system and the bottom panel in the homologous hCG- $\beta$ system, respectively. Results are expressed in terms of the logit of percent bound $v s$ the log of the dose. respective inhibition line parallel to that of purified hCG in the homologous hCG system, parallel to that of purified hCG- $\alpha$ in the homologous hCG- $\alpha$ system and parallel to that of purified hCG- $\beta$ in the homologous hCG- $\beta$ system (Fig. 3). The void-volume material was recognized as being $0.67 \%$ as potent as purified hCG, $0.1 \%$ as potent as purified hCG- $\alpha$ and $0.33 \%$ as potent as purified hCG- $\beta$, respectively.

\section{Tryptic digestion}

The susceptibility of the immunoreactive void-volume material to be converted to hCG by tryptic digestion was investigated by incubating the void-volume material with trypsin. As shown in Fig. 4, although refiltration of the undigested sample showed

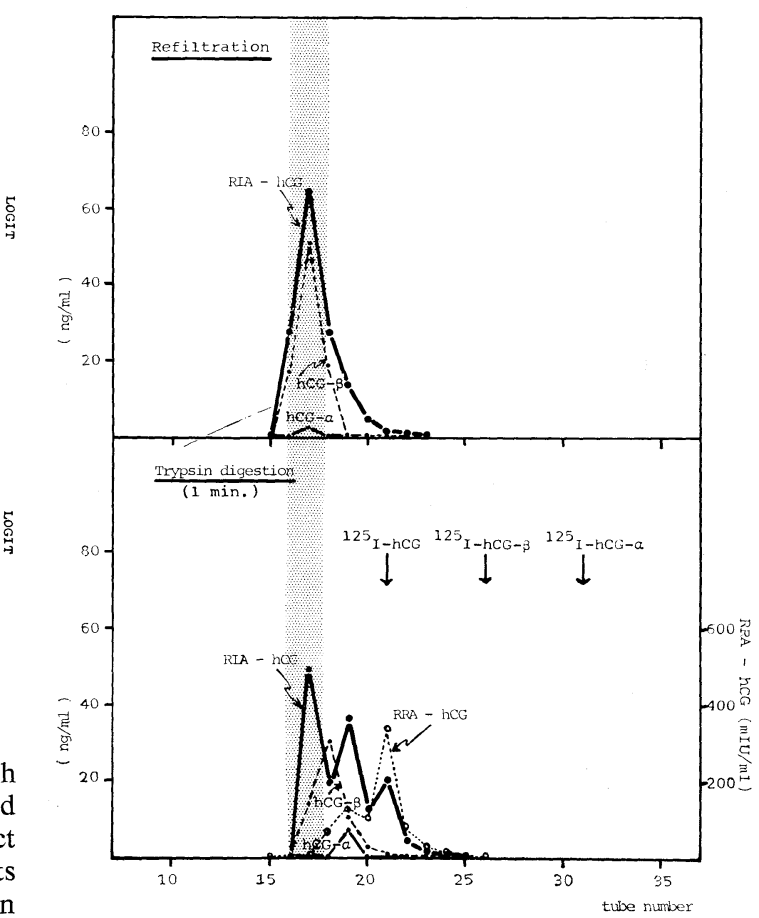

Fig. 4. Elution profile of the immunoreactive voidvolume material $(400 \mu \mathrm{g})$ before and after trypsin exposure for $1 \mathrm{~min}$ from Sephadex G-100 column. Top panel: before trypsinization, and bottom panel: after trypsinization. 
that immunoreactivity of $\mathrm{hCG}, \mathrm{hCG}-\alpha$ and $\mathrm{hCG}-\beta$ was recovered entirely in the void volume, the elution profile of the trypsin digested sample was considerably changed, with three different peaks of hCG immunoreactivity. The first hCG peak was present in the void volume. The second hCG peak was apparently another larger molecular species. The most retarded hCG peak appearing in the region of ${ }^{125} \mathrm{I}-\mathrm{hCG}$ was accompanied with biological activity. No conversion of the void-volume material to the authentic form of hCG- $\alpha$ and hCG- $\beta$, however, was observed by the tryptic digestion.

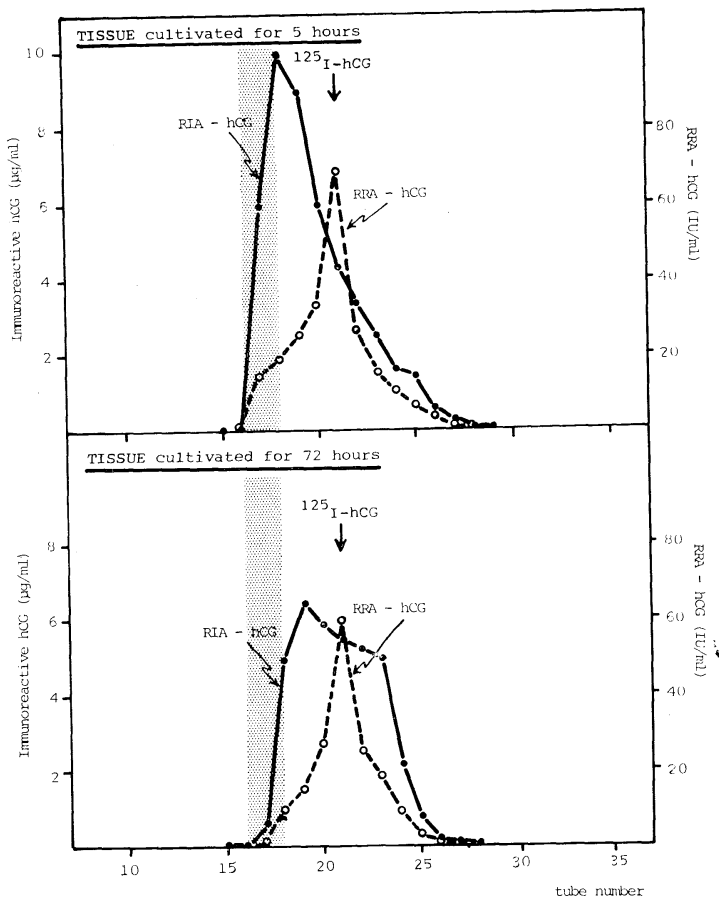

Fig. 5. Elution profiles of immunoreactive hCG and bioactive hCG in extracts of the chorionic tissues cultivated in vitro from Sephadex G-100 column. Top panel: chorionic tissue after 5-hr culture, and bottom panel: chorionic tissue after 72-hr culture. Immunoreactive hCG was determined by homologous radioimmunoassay for hCG and bioactive hCG by the radioreceptor assay using membrane-bound receptor fraction of Parlow rat ovary.
Heterogeneity of $h C G$ with respect to $\mathrm{im}$ munoreactivity and biological activity

In order to search for the heterogeneity of hCG with respect to immunoreactivity and biological activity, each eluate on Sephadex gel filtration of extracts of cultivated chorionic tissues and culture media was measured by the radioimmunoassay and radioreceptor assay. The elution profiles of the extracts of chorionic tissue cultivated in vitro are illustrated in Fig. 5. The elution peak of hCG immunoreactivity demonstrated a shift away from the void volume to the more retarded area in relation to the increased time of culture and

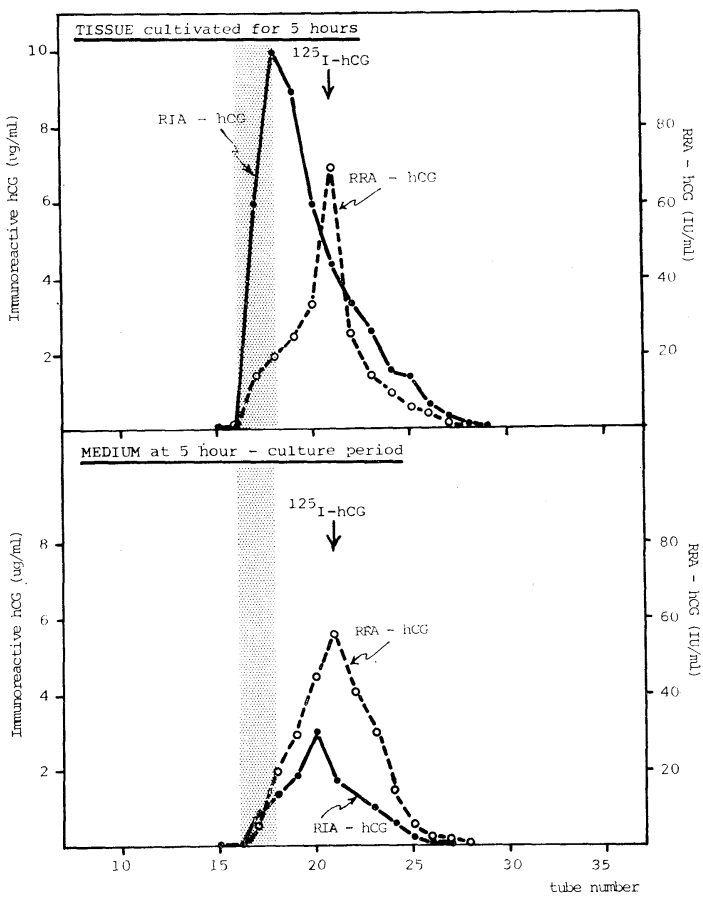

Fig. 6. Elution profiles of immunoreactive hCG and bioactive hCG in the chorionic tissue cultivated in vitro for $5 \mathrm{hr}$ and in its media from Sephadex G-100 column. Top panel: in the placental extract after 5 -hr culture, and bottom panel : in the media after 5 -hr culture. Immunoreactive hCG was determined by the homologous radioimmunoassay and bioactive hCG by the radioreceptor assay using membrane-bound-receptor fraction of Parlow rat ovary. 
a "shoulder" of immunoreactive hCG was simultaneously apparent on the right side of the peak, exhibiting molecular heterogeneity of immunoreactive hCG. In contrast, the elution peak of bioactive $\mathrm{hCG}$ determined by the radioreceptor assay was always apparent in the region of urinary purified hCG labeled with ${ }^{125} \mathrm{I}$ used as a column marker without exhibiting heterogeneity. Within the chorionic tissues cultivated in vitro, the amount of immunoreactive hCG was much larger than that of bioactive hCG since biological potency of purified $\mathrm{hCG}$ used in this study was $15,000 \mathrm{IU} / \mathrm{mg}$ by an ovarian weight method.

Fig. 6 displays comparison of the elution profile of the extract of chorionic tissue cultivated for $5 \mathrm{hr}$ with that of the culture medium. After filtration of the medium, both peaks of immunoreactive hCG and bioactive hCG were apparent near the region of ${ }^{125} \mathrm{I}-\mathrm{hCG}$. A large amount of bioactive $\mathrm{hCG}$ was found within the 5-hr culture medium, relative to the amount of immunoreactive $\mathrm{hCG}$.

\section{Discussion}

Reports from several laboratories, including our own, have demonstrated heterogeneity of glycoprotein hormones and their subunits. Evidence for the large species of luteinizing hormone ( $\mathrm{LH})$ in the pituitary has been reported by Reichert (1965) and Prentice and Ryan (1975). Vaitukaitis (1974) and Maruo (1976) have described the existence of the large immunologic species of hCG in the placenta. There has been, however, no report concerning the characterization of the large immunologic species of hCG which appeared in the void volume of the Sephadex G-100 column. The present study infers some of the characteristics of the immunoreactive voidvolume material. The immunoreactive voidvolume material is not a simple aggregate of hCG or an association of hCG with another protein for the following reasons; a) The immunoreactive void-volume material does not dissociate into hCG when it is refiltered on Sephadex. b) Following a short exposure to $5 \mathrm{~m}$ guanidine, elution position of hCG immunoreactivity is unchanged and a slight reduction in total hCG immunoreactivity is observed accompanied with dissociation into hCG- $\alpha$ and hCG- $\beta$ subunits larger than urinary purified hCG- $\alpha$ and hCG- $\beta$. These observations suggest that the immunoreactive void-volume material might contain "big hCG" and it could be dissociated into large molecular species of hCG- $\alpha$ and hCG- $\beta$ by guanidine treatment. Immunoreactive hCG- $\alpha$-like forms of apparently the larger molecular size in gel filtration have been noted in placental extracts (Vaitukaitis, 1974; Maruo, 1976) and in a gastric carcinoid tumor extract (Weintraub et al., 1975). Although the biological significance of these large immunologic species of hCG subunits is not yet known, Weintraub et al. (1975) speculated on a possibility that the large immunologic species of hCG- $\alpha$ might represent a precursor form of the authentic form of hCG- $\alpha$.

In immunologic studies, the immunoreactive void-volume material and purified hCG showed complete immunologic crossreactivity in the homologous hCG assay system with the slope of inhibition line being equal to each other. However, the 150 -fold greater mass was required for comparable inhibition of the immunoreactive void volume material, relative to purified hCG. That difference may reflect the ratio of the immunoreactive hCG to the total protein contents within the cultivated chorionic tissues, since the void-volume material is a crude preparation obtained by the initial gel filtration on Sephadex G-100. It was previously described in our preliminary report (Maruo et al., 1974) that the ratio of the immunoreactive $\mathrm{hCG}$ to the total protein contents within the cultivated 
chorionic tissues was $0.0028-0.0046$ during the 3-day culture. These results suggest that "big hCG" in the immunoreactive void volume material might be immunologically indistinguishable from purified hCG. On the other hand, it was simultaneously demonstrated that the void volume material might have common immunologic determinant with purified hCG- $\alpha$ and purified hCG- $\beta$. It is possible that "big hCG- $\alpha$ " and "big hCG- $\beta$ " also might exist in the immunoreactive void-volume material.

The possibility that the immunoreactive void-volume material represented a prohormone of hCG was investigated by tryptic digestion in an attempt to convert it to hCG. Tryptic digestion of the immunoreactive void-volume material resulted in an appearance of biologically active hCG which was eluted in the region of ${ }^{125} \mathrm{I}$-hCG. The elution pattern of hCG immunoreactivity from Sephadex G-100 was, however, heterogeneous. It is possible that the time of digestion was too short to obtain the necessary cleavage or that some other proteolytic enzyme might be required for the complete conversion of the immunoreactive volid volume material. These results are suggestive of a possible precursor role of "big hCG" within the void-volume material in the biosynthetic process of $\mathrm{hCG}$, as the precursor relationship of proinsulin to insulin. However, opinions about the necessity of a prohormone for identical biosynthesis of glycoprotein hormone are a bit confusing. Prentice and Ryan (1975) have demonstrated the possible existence of the void-volume material with immunologic $\mathrm{LH}$ activity in pituitary extracts and have offered a hypothesis that LH is synthesized as a prohormone. In contrast, Pierce et al., (1976) suggested from the results of the experiments on reoxidation of reduced subunits of LH that a prohormone is not necessary for the initial biosynthesis of glycoprotein hormones. Confirmation of the necessity of a prohormone for hCG biosynthesis still awaits demonstration of the appearance of authentic hCG to which the major radioactive peak shifts by tryptic digestion of "big hCG" incorporporated radioactive amino acid. We have not yet confirmed such a shift of radioactive peak as noted above in the experiment of tryptic digestion of "big hCG".

Heterogeneity in the molecular size of bioactive hCG was not found by the radioreceptor assay. HCG radioreceptor activity following the gel filtration of cultivated chorionic tissue extracts and of media was always eluted with a single peak in the region of ${ }^{125} \mathrm{I}-\mathrm{hCG}$. This observation indicated that the receptor activity of authentic hCG is high but that of "big hCG" was diminished. On the other hand, as shown in RESULTS, the authentic form of hCG might be a predominant form of the hormone in the culture media, whereas the large immunologic species of hCG might be a predominant form in the cultivated chorionic tissues. In fact Tojo et al. (1974) previously reported that hCG immunoreactivity in the chorionic tissue was about 12-fold greater than hCG bioactivity detected by an ovarian weight method and that there was a marked excess secretion of bioactive hCG, relative to immunoreactive hCG into the media with the increased time of culture. In detail, the amount of bioactive hCG in the media after 5-day culture was estimated to be about 40 -fold greater than that in the chorionic tissue prior to culture, whereas the amount of immunoreactive hCG in the media was only about 2-fold greater than that in the chorionic tissues prior to culture. These results infer that the culture media rich in authentic form of hCG contain high hCG bioactivity while the chorionic tissues rich in large immunologic species of hCG contain higher immunoreactivity in comparison to bioactivity. These findings may be somewhat related with the observation of Ashitaka (1970), Flury and Martin (1972) and Pala et al. (1973) who have demon- 
strated that the biological potency of the purified hCG obtained from placenta is much lower than that of the purified hCG obtained from urine of pregnant women.

The present studies do not permit us further to define the nature of the heterogeneous immunoreactive forms of $\mathrm{hCG}$ and its extracts. Additional studies on the in vitro biosynthesis of the large immunologic species of hCG and its subunits will be necessary to elucidate the in vitro interrelationship of these heterogeneous forms.

\section{References}

Ashitaka, Y. (1970). Acta Obst. Gynec. Japon. 17, 124. Ashitaka, Y., Y. Tokura, M. Tane, M. Mochizuki and S. Tojo (1970). Endocrinology 87, 233.

Ashitaka, Y. and S. S. Koide (1974). Fertil. Steril. 25, 177.

Ashitaka, Y., R. Nishimura, Y. Endoh and S. Tojo (1974). Endocrinol. Japon. 21, 429.

Flury, A. and A. Martin (1972). Hoppe-Seylers $Z$.
Physiol. Chem. 353, 1401.

Lee, C. Y. and R. J. Ryan (1973). J. Clin. Endocrinol. Metab. 36, 148.

Maruo, T., Y. Ashitaka, M. Mochizuki and S. Tojo (1974). Endocrinol. Japon. 21, 499.

Maruo, T. (1976). ibid. 23, 119.

Pala, A., M. Meirinho and G. Benagiano (1973). J. Endocr. 56, 441.

Pierce, J. G.. M. R. Faith, L. C. Giudice and J. R. Reeve. Polypeptide Hormones: Molecular and Cellular Aspects. Ciba Foundation Symposium \#41., (edited by R. Porter and D. W. Fitzsimons). Excerpta Medica, Amsterdam, p. 225 (1976).

Prentice, L. G. and R. J. Ryan (1975). J. Clin. Endocrinol. Metab. 40, 303.

Reichert, L. E. Gonadotropins: Physicochemical and Immunological Properties, (edited by G. Wolstenholme and J. Knight). Ciba Foundation Study Group $\$ 22$, Little, Brown \& Co., Boston (1965).

Tojo, S., M. Mochizuki and T. Maruo. Gonadotropins and Gonadal Function, (edited by N. R. Moudgal). Academic Press, New York, p. 321 (1974).

Vaitukaitis, J. L. (1974). J. Clin. Endocrinol. Metab. $38,755$.

Weintraub, B. D., G. Krauth, S. W. Rosen and A. S. Rabson (1975). J. Clin. Invest. 56, 1043. 